\title{
The Dynamical Approach as Practical Geometry
}

February 28, 2014

\begin{abstract}
This essay introduces Harvey Brown and Oliver Pooley's 'dynamical approach' to special relativity and argues that it is best construed as a relationalist form of Einstein's 'practical geometry', according to which Minkowski geometrical structure supervenes upon the symmetries of the best-systems dynamical laws for a material world with primitive topological or differentiable structure. This construal of the dynamical approach is shown to be compatible with the related chapters of Brown's text, as well as recent descriptions of the dynamical approach by Pooley and others.
\end{abstract}

Total Word Count: 5,022 


\section{Contents}

$\begin{array}{lll}1 & \text { An Introduction to the Dynamical Approach } & 1\end{array}$

2 Ideological Relationalism and the Constructive Project 4

\begin{tabular}{|lll}
3 & Einstein's 1921 Geometry and Experience & 6
\end{tabular}

4 On Rigid Bodies and Practical Geometry

$\begin{array}{lll}5 & \text { Practical, Supervenient Geometry } & 12\end{array}$

$\begin{array}{lll}6 & \text { Brown's Coordinatization Project } & 15\end{array}$

\begin{tabular}{lll}
\hline 7 & Conclusions & 18
\end{tabular} 


\section{An Introduction to the Dynamical Approach}

In Physical Relativity (2005), Harvey Brown promotes an unorthodox interpretation of special relativity, which shall be referred to here as the 'dynamical approach'. In brief, the dynamical approach argues for the priority of dynamics over geometry in the context of explanation. Relativistic kinematical phenomena like length contraction and time dilation, along with inertial motion and even Minkowski geometrical structure, are to be explained by the fact that the dynamical laws governing the behavior of material objects are Lorentz-covariant. ${ }^{2}$ In addition to these explanatory claims, it has been pointed out by others that if the symmetries of the dynamical laws are to somehow explain Minkowski geometry, then there is also an implicit ontological claim being made. Indeed, this ontological claim is evident in the title of Brown's collaboration with Oliver Pooley: Minkowski Space-Time, a Glorious Non-Entity (2006). That is, while the primary focus of Brown's work is not the ontological status of spacetime, the dynamical approach to

\footnotetext{
${ }^{1}$ In an earlier collaboration with Pooley (Brown and Pooley 2001), the dynamical approach was introduced as 'the truncated Lorentzian pedagogy'. This and their later collaboration (2006) are subsumed in Brown's Physical Relativity (2005). For simplicity, I refer primarily to Brown, and quote primarily from Physical Relativity, when introducing their collaborative promotion of the dynamical approach.

${ }^{2}$ See Physical Relativity (pp.vii-viii, pp.24-5, ch.8). Note that Brown and Pooley distinguish the dynamical approach from a 'fully constructive' theory, which would offer explanations that appeal to the details, rather than to the symmetries, of the dynamical laws (2006, p.82). They also distinguish it from a neo-Lorentzian approach, which would advocate the existence of a preferred frame (2001, p.257).
} 
relativity is arguably best construed as a form of relationalism, according to which spacetime does not exist primitively. For otherwise, it could not be said that the symmetries of the dynamical laws somehow account for its geometrical structure. ${ }^{3}$ But more importantly, it should also be pointed out that this explanatory claim comes with an implicit ideological claim: that material objects' primitive properties do not constitute a Minkowskian geometrical structure. ${ }^{4}$ For instance, according to the dynamical approach, material objects cannot stand, primitively, in the same chronometric relations that they would if existing in substantival Minkowski spacetime. Otherwise, it could not be said that the symmetries of the dynamical laws somehow account for the material world's Minkowskian geometrical structure. Rather, the latter would be constituted by the fundamental entities' primitive chronometric relations; the fundamental entities would instantiate Minkowski geometry on their own. This would not do for the dynamical approach, which describes that geometrical structure as "parasitic on the relativistic properties of

\footnotetext{
${ }^{3}$ As mentioned below, Pooley describes the dynamical approach as a form of relationalism in later work $2013, \$ 6.3 .2)$. However, so long as kinematical effects and inertial motion are explained by an appeal to the symmetries of the dynamical laws (rather than those of spacetime), the dynamical approach would actually be compatible with a form of substantivalism according to which spacetime has less geometrical structure than that which best codifies the behavior of material objects. This is addressed further in footnote 23 .

${ }^{4}$ Following Quine (1951), the ontology lists the entities to whose existence the theory is committed, and the ideology lists those entities' primitive properties or relations.
} 
the dynamical matter fields" and as "no more than a codification of the behavior of rods and clocks" (Brown 2005, p.9, p.100). ${ }^{5,6}$ Thus of the three explanatory claims listed above, the idea that dynamics explains geometry is what first associates the dynamical approach with relationalism, and that also illustrates the difference between the dynamical approach and a more 'traditional' relationalist approach: in the latter, the material world's spatiotemporal geometry is constituted by material objects' primitive properties; in the former, it supervenes upon the symmetries of the dynamical laws. ${ }^{7}$

${ }^{5}$ See also (Brown 2005, ch.8), especially pp.142-3.

${ }^{6}$ Note that Brown is not idealizing rods and clocks as privileged entities in the way Einstein did (1905) and later regretted (1949a pp.59-61). Rather, Brown understands rods and clocks to be atomic (or field) configurations governed by Lorentz-covariant dynamical laws 2005, p.4). That is, he takes them to be solutions of the dynamical equations, and he takes the equations' Lorentz-covariance to ensure that the behavior of rods and clocks is encoded by Minkowski geometry.

${ }^{7}$ The viability of such a 'traditional' relationalist approach to Minkowski spacetime structure is explored by Earman $(1989, \S 6.10)$, Maudlin $(1993, \S 5)$, and Pooley (2013, §6.1; 2014, §5). 


\section{Ideological Relationalism and the Constructive Project}

The dynamical approach has received a number of responses in the literature. For the most part, these have focused on the explanatory claims mentioned above. ${ }^{8}$ Some, however, have focused primarily upon the implicit ontological claim mentioned above. ${ }^{9}$ Insofar as the two can be decoupled, this essay will focus primarily upon the latter, with an emphasis on the aforementioned ideological claim.

The question at hand is this: if Minkowskian geometrical structure is to supervene upon the behavior of material objects that act in accord with Lorentz-covariant dynamical laws, then in what kind of primitive spatiotemporal relations could those material objects stand, or what kind of primitive spatiotemporal structure could they instantiate? From just the brief description above, it should be clear that chronometric 'interval' relations cannot feature in the list of material objects' primitive properties (i.e. in the 'ideology'). Similarly, because the causal structure of Minkowski geometry determines its metrical structure up to scale, causal relations are also banned from the ideology. ${ }^{10}$ Thus the dynamical approach must be

${ }^{8}$ For example: Balashov and Janssen (2003); Janssen (2009); Dieks (2009); Dorato and Felline (2010); Felline (2011); Frisch (2011); Lange (2011); van Camp (2011). Many of these (among others) also focus upon Einstein's claims about the difference in explanatory power of 'principle' and 'constructive' theories.

${ }^{9}$ The implicit ontological claim is acknowledged by Huggett (2009) and by Pooley (2013). It is emphasized by Nerlich (2013), and especially by Norton (2008). ${ }^{10}$ Earlier work on this by Robb 1914,1936$)$ and Zeeman $(1964)$ is brought together nicely by Winnie (1977). 
what might be called an ontologically and ideologically relationalist approach to Minkowski geometry: it must provide a material ontology that excludes Minkowski spacetime, and it must provide a spatiotemporally scant ideology, the spatiotemporal components of which do not constitute Minkowski geometry. Then, with respect to some such metaphysics, it must be shown that Minkowski geometry is derivative of facts about the symmetries of the dynamical laws.

This ideologically relationalist aspect of the dynamical approach is what has led some to refer to it as 'constructive relativity'. In recent work, John Norton describes the 'constructivist project' as the task of inferring Minkowski geometry "from the properties of matter without recourse to spatiotemporal presumptions or with few of them" (2008, p.821). And the key word here is "few", for the explanatory claims of the dynamical approach certainly do not require that all geometrical structures or concepts supervene upon the symmetries of the dynamical laws. As Pooley has recently noted, "the project was to reduce chronogeometric facts to symmetries, not to recover the entire spatiotemporal nature of the world from no spatiotemporal assumptions whatsoever" 2013, p.573). And so, what the constructivist project actually involves is the specification of some primitive spatiotemporal structure which, while not constituting Minkowski geometry itself, could somehow support the use of Lorentz-covariant laws in systematizing material objects' degrees of freedom. This would seem to require that the dynamical laws be written in terms of more geometrical structure than is ascribed as primitive of the material world, and it will be argued below that this commitment gives the dynamical approach a unique position in the empiricist-conventionalist spectrum of traditional approaches to geometrical structure.

To begin, Einstein's well-known contrast between empiricism and convention- 
alism is outlined in $\$ 3$, and what appear to be his own views are distinguished from the former two in $\$ 4$. It is then argued in $\$ 5$ that the dynamical approach is best understood as an adaptation of Einstein's perspective, according to which Minkowski geometry supervenes upon the behavior of rods and clocks.

\section{Einstein's 1921 Geometry and Experience}

The idea that spacetime geometry is an empirical issue, and that it should therefore be described as a branch of physics, was popularized by Einstein in a lecture before the Prussian Academy of Sciences, which was published under the title Geometry and Experience in the same year (1921). In the opening paragraphs, Einstein makes an important distinction between what he calls 'pure' or 'axiomatic' geometry and 'practical' geometry. ${ }^{11}$ The former is Einstein's name for the formal, axiomatic mathematics itself, which consists of primitive terms that are defined implicitly by the axioms they satisfy. ${ }^{12}$

[Pure] geometry treats of objects which are denoted by the words straight line, point, etc. No knowledge or intuition of these objects is assumed, but only the validity of the axioms, $[\ldots]$ which are to be taken in a purely formal sense, i.e. as void of all content of intuition or

\footnotetext{
${ }^{11}$ Einstein makes the same distinction elsewhere (1925). Carl Hempel also gives a similar introduction (1945). Note that some authors call the former 'mathematical' geometry, and the latter 'physical' or 'applied' or 'empirical' geometry.

${ }^{12}$ It should be noted that this understanding of geometry comes originally from David Hilbert (1971).
} 
experience.

(1921, p.234)

On this view, pure geometry can make no empirical claims. Instead, its value lies in the certainty of its mathematical claims.

Practical geometry, on the other hand, is what results from making some coordination between material objects and the primitive terms of pure geometry. With some such coordination in place, the logical deductions of pure geometry can be translated into hypotheses about the behavior of material objects. In this way, spacetime geometry becomes a branch of physics.

It is clear that the system of concepts of axiomatic geometry alone cannot make any assertions as to the behavior of real objects of this kind, which we will call practically-rigid bodies. To be able to make such assertions, geometry must be stripped of its merely logical-formal character by the coordination of real objects of experience with the empty conceptual schema of axiomatic geometry. To accomplish this, we need only add the proposition: solid bodies are related, with respect to their possible dispositions, as are bodies in Euclidean geometry of three dimensions. Then the propositions of Euclid contain affirmations as to the behavior of practically-rigid bodies. [...] The question whether the practical geometry of the universe is Euclidean or not has a clear meaning, and its answer can only be furnished by experience.

(1921, pp.234-5)

However, the price of some such coordination is that the certainty of pure geometry is lost in the applied, practical geometry; the same experience that provides answers to the question of the material world's geometrical structure is also what spoils the purity of those answers. Hence Einstein's claim that "as far as the propositions of 
mathematics refer to reality, they are not certain; and as far as they are certain, they do not refer to reality" (p.233).

Now, Einstein's purposes in making the distinction are clear: it comes as part of his response to a certain epistemological question about geometry - namely, the question of whether geometry can provide us with synthetic a priori knowledge. At the time of Einstein's writing, advocates of the neo-Kantian tradition held that it could, and Einstein's response has typically been taken as illustrating his ties with logical positivism. For until some coordinations are made between the pure geometrical primitives and the material world, 'pure' geometry itself provides no information about the material world. ${ }^{13}$

Whatever Einstein's position on the epistemic question, he has emphasized more than once that without this conception of geometry, he could not have developed general relativity (1921, p.235). ${ }^{14}$ But the fact that Einstein found it so useful does not make it uncontroversial. Later in the same article, Einstein goes on to introduce a rival, conventionalist view of geometry, which is best known by its promotion in the work of Henri Poincaré $(1898$; 1905). In short, Poincaré rejects the coordination of pure geometry's primitive terms with material objects, and his reasons are clear: Einstein's coordination involves rigid bodies, of which there are none. Any potential candidates - Einstein refers to "measuring lines, measuring wands, etc." as examples (p.234) - are subject to conditions like temperature and pressure, to a degree that would be specified by a theory of matter. What's more, even when those influences are accounted for by a matter theory, any talk of rigidity would be

\footnotetext{
${ }^{13}$ Friedman (2002), Giovanelli (2012), and Howard (2014) discuss this further.

${ }^{14}$ See also the references provided by Friedman (2002, p.207ff).
} 
made in relation to some geometry, meaning that a geometrical structure would have been assumed already. As Einstein explains, Poincaré's denial of the necessary coordination eliminates the possibility of 'practical' geometry at all, and leads to the view that spacetime geometry is not an empirical issue, but a conventional one (p.236ff). And were the choice up to him, Poincaré would prefer to use Euclidean geometry over anything more complicated or unfamiliar.

\section{On Rigid Bodies and Practical Geometry}

At this point, Einstein has presented two incompatible perspectives on geometrical structure. Given his testimony about the usefulness of 'practical geometry', it would seem that he takes the empiricists' side. ${ }^{15}$ But in the same paper, Einstein admits to taking sides with Poincaré, at least in principle.

Sub specie aeterni Poincaré, in my opinion, is right. The idea of the measuring-rod and the idea of the clock coordinated with it in the theory of relativity do not find their exact correspondence in the real world. It is also clear that the solid body and the clock do not in the conceptual edifice of physics play the part of irreducible elements, but

\footnotetext{
${ }^{15}$ Einstein made similar claims when writing on the same topic in the years immediately following $(1924 ; 1925)$, and later attributed the position to Hans Reichenbach (1949b, p.679). Indeed, the empiricist approach that Einstein advocates above is very much in line with that of Reichenbach 1 1949; 1958), whose use of 'coordinative definitions' establishes a link between 'axiomatic' and 'empirical' geometry. Closer comparisons are offered by Giovanelli (2012) and Howard (2014).
} 
that of composite structures, which must not play any independent part in theoretical physics. [...] But it is my conviction that in the present stage of development of theoretical physics these concepts must still be employed as independent concepts; for we are still far from possessing such certain knowledge of the theoretical principles of atomic structure as to be able to construct solid bodies and clocks theoretically from elementary concepts.

$$
\text { 1921, pp.236-7; cf. 1949b, pp.676-9) }
$$

Taking this statement into consideration, it would seem that Einstein, while fully endorsing the methodology of 'practical geometry', would abstain from committing himself to the existence of rigid bodies. But of course, to do so would be to deny that geometrical structure is in fact a branch of science. Recognizing this, Einstein follows the quotation above with a clarification that the physical state of a measuring body can always be determined "so accurately that its behavior relative to other measuring-bodies shall be sufficiently free from ambiguity to allow it to be substituted for the 'rigid' body", and that "it is to measuring-bodies of this kind that statements about rigid bodies must be referred" (1921, p.237). So long as these 'practically' rigid bodies are taken to exist, Einstein would still view geometry as something to be determined empirically. But to go any further in denying the existence of rigid bodies would lead to a very different perspective. In another popular-level article on a similar topic, Einstein expounds upon this point. $^{16}$

\footnotetext{
${ }^{16}$ Another translation is given by Howard $(2014$, p.20). Einstein also makes a very similar point elsewhere $(1925)$.
} 
The stance on these theses depends on whether one grants reality to practical rigid bodies. If yes, then the concept of distance corresponds to something experienceable. Geometry then contains statements about possible experiments; it is a physical science directly subject to experimental proof (standpoint A). If no reality is conceded to practical rigid measurable bodies, the geometry alone holds no statements about experiences (experiments), rather just geometry together with the physical sciences (standpoint B). Physics has hitherto always used the simpler standpoint A and largely owes to it its productivity; it uses it in all its measurements. [...] If, however, one assumes standpoint B, which at the current state of science should be regarded as exaggeratedly cautious, then geometry on its own is not experimentally testable. Then geometrical measurements don't exist at all. [...]

Seen from standpoint B, the selection of geometric concepts and relations is determined only for reasons of simplicity and practicality. Under no condition does the choice of a non-Euclidean geometry presuppose Euclidean geometry as its basis. But then nothing can be discerned empirically about the dimensions of the space, but not because "space is not real," rather because with this choice of standpoint geometry is not a complete physical system of concepts but only part of one.

(1924, p.202)

Now, in order to make sense of Einstein's claim that standpoint B should be regarded as "exaggeratedly cautious," it should be kept in mind that standpoint A does not involve a commitment to the existence of rigid bodies, but only practically rigid bodies. Thus standpoint A is effectively Einstein's position, even after agreeing 
with Poincaré in principle, as outlined in his 1921 lecture. But here a clarification is in order: by granting existence only to practically rigid bodies, Einstein's 'practical geometry' loses at least some of its punch. In that case, it could not be said that the primitive geometrical structure of the material world can be determined empirically, but merely that, to good approximation, material objects behave in accord with some particular geometrical structure.

\section{Practical, Supervenient Geometry}

We now return to the question of where the dynamical approach could fit into the empiricist-conventionalist divide. To begin, it should be pointed out that if emphasis is placed on the explanatory claims of the dynamical approach, then one might be led to think that Brown is advocating empiricism. Along these lines, Bradford Skow shows us how far the comparison could be taken.

Maybe Brown thinks that the symmetries of the laws explain why spacetime has the structure it does because the spacetime metric is somehow analyzed in terms of the laws. In several places his discussion suggests that, on his view, a field only gets to be the metric field when the dynamics prescribe that material bodies behave in the right sort of way with respect to it (see, for example, pages 100 and 160). Compare this to a Reichenbachian view according to which distance between points of space is analyzed in terms of the behavior of measuring rods - though Brown rejects this view (23), his view does seem to resemble it: both of them make spacetime geometry an extrinsic matter. (Brown's apparent denial that spacetime geometry is "self-standing" 
and "autonomous" looks like a denial that it is intrinsic (143).)

$$
\text { (2006, p.4) }
$$

As Skow notes, Brown seems to reject the 'practical geometry' approach in its strongest form, according to which the existence of rigid bodies paves the way for the world's primitive geometry to be determined empirically. After all, Brown is careful to treat rods and clocks as complex entities governed by dynamics, and not as privileged entities that could play a role in the coordination process outlined above. ${ }^{17}$ Indeed, given Brown's emphasis on Einstein's misgivings about privileging rods and clocks in the development of special (and general) relativity, his work might even be taken as supportive of Poincaré's conventionalism-but for the fact that Brown advocates Minkowskian rather than Euclidean geometry, and speaks freely about the symmetries of dynamical laws without assuming some geometrical structure first.

Thus Brown's work cannot easily be read as a support of either 'ism' outlined in $\$ 3$. And nor should it. The strict empiricist perspective would not support the explanatory claim in which symmetries of the dynamical laws account for Minkowski geometry, but only that those symmetries can reveal to us the world's primitive spatiotemporal structure. And already it has been explained that the dynamical approach is not compatible with an ideology in which Minkowski geometrical structure is taken as primitive. On the other hand, Poincaré's conventionalist approach would require an argument in favor of Minkowski geometry on the basis of its simplicity relative to other geometrical structures. And even if such an argument could be made, it would get us nowhere in accounting for that geometry

\footnotetext{
${ }^{17}$ See earlier comments in footnote 6 , above.
} 
by appealing to the symmetries of dynamical laws. Instead, the dynamical approach seems to land much closer to Einstein's own views, according to which truly rigid bodies are not assumed, and yet the behavior of material bodies plays a substantive role in arriving at some particular spacetime structure. The crucial difference, of course, is that the dynamical approach's arrow of explanation from dynamics to geometry would require that the geometrical structure determined by the methods of 'practical geometry' should not be taken as a primitive feature of the world, but as somehow supervening upon the behavior of material objects themselves.

To see how such a project might succeed, recall from above $(\$ 2)$ that the dynamical approach is not prohibited from assuming some kind of sub-metrical spatiotemporal structure, so long as the full structure of Minkowski spacetime is still somehow picked out by the symmetries of the dynamical laws. Although the technical details of such an approach are beyond the scope of this paper, the general scheme could be completed by taking a particular Humean conception of natural laws, according to which regularities in the 'Humean mosaic' may be described with the aid of more geometrical structure than is actually present in the mosaic. ${ }^{18}$ And if, for a particular Humean mosaic, the best-systems dynamical laws are in fact written in terms of some such supplementary geometrical structure, then that structure would supervene upon the symmetries of the dynamical laws. To put it in other words, a material system with sub-metrical spatiotemporal structure could always be labeled by sets of coordinates that are related to one another by transformations corresponding to the symmetry group of a richer geometrical

\footnotetext{
${ }^{18}$ This 'liberalized' Humean conception of dynamical laws is introduced by Huggett $(2006)$, and is discussed further in $\$ 7$ below.
} 
structure. And if some such set of coordinatizations are the ones with respect to which the best-systems dynamical laws are written, then that set is used in determining the mosaic's supervenient geometrical structure.

In this way, a world in which rigid bodies clearly do not exist is still a world in which the methods of Einstein's practical geometry can be applied. In the process, the dynamical approach's arrow of explanation from dynamics to geometry is vindicated: the primitive spatiotemporal structure of the material world is sub-metrical, while Minkowski geometry supervenes upon the symmetries of the best-system dynamical laws.

\section{Brown's Coordinatization Project}

In the opening of the second chapter of Physical Relativity, Brown raises a question that occupies his attention for the remainder of that section:

It is common in discussions of the principle of general covariance in Einstein's general theory of relativity to find the claim that coordinates assigned to events are merely labels. [...] Before we consider labeling them, what physically distinguishes two different events of exactly the same kind? (2005, p.11)

Brown's response reveals that he has in mind an ontology of physical fields with some kind of sub-metrical structure.

Brown begins his response by casting doubt upon the existence of points of substantival spacetime. He reviews Robert Geroch's (1978) distinction between a localized 'material event' - like a firecracker or finger-snapping, and which Brown calls a physical 'marker' - and the point of spacetime in/at which the event is 
taken to occur in a substantivalist perspective. And whereas Geroch was allegedly hesitant to make a distinction between the 'point' and its 'marker', Brown is clear in stating that he does not distinguish the two at all. That is, Brown is not interested in the relationships between points of spacetime, but rather in the relationships between their markers. Thus an ontological relationalism is suggested, even if it is not crucial to Brown's thesis. ${ }^{19}$ And in the place of substantival spacetime points, Brown suggests a fundamental ontology of 'markers' (material events) that comprise coincident values of physical fields. ${ }^{20}$

Then, Brown's ideology is revealed by his approach to distinguishing such material events for the purposes of coordinatization. ${ }^{21}$ As Brown explains, there will surely exist various types of material events that are intrinsically indiscernible (2005, p.13). And at this point, Brown finds himself in a more difficult situation than the traditional relationalist. If Brown were to admit Minkowski intervals as primitive relations between material events, then indistinguishable material events would not be an issue for any but the most unrealistically homogeneous or symmetric universes. Rather, the possible coordinatizations would be fixed by the requirement that they respect the structural properties that supervene upon the field values' spatiotemporal relations. ${ }^{22}$ But so far, Brown has only admitted

\footnotetext{
${ }^{19}$ See footnotes 3 and 23 .

${ }^{20}$ See (Brown 2005, p.13). This is consistent with what Brown has written
} elsewhere (1997, p.68). A similar point has been made by Pooley $(2014, \S 5.8)$.

${ }^{21}$ In the spirit of the physicists to whom Brown refers, I refer to Geroch's events as 'points of substantival spacetime' and to their markers as 'material events'.

${ }^{22}$ See (Pooley 2014, §5.8) for a discussion. 
material events, and in order to justify any particular choice of coordinatization, he must begin to build up an appropriately rich spatiotemporal ideology without undermining the goal of accounting for metrical structure in terms of the symmetries of dynamical laws.

Toward that end, Brown argues that each individual material event stands in a unique relationship to the universe as a whole. That is, material events "have different coordinates because they are distinct, and they are distinct not in virtue of what they are locally but in virtue of the fact that they stand in different relations to the rest of the universe - to the rest of the markers" (p.14). To illustrate, Brown borrows a phrase from Julian Barbour (1982).

Minkowski, Einstein, and Weyl invite us to take a microscopic look, as it were, for little featureless grains of sand, which, closely packed, make up space-time. But Leibniz and Mach suggest that if we want to get a true idea of what a point of space-time is like we should look outward at the universe, not inward into some supposed amorphous treacle called the space-time manifold. The complete notion of a point of space-time in fact consists of the appearance of the entire universe as seen from that point. Copernicus did not convince people that the earth was moving by getting them to examine the earth but rather the heavens. Similarly, the reality of different points of space-time rests ultimately on the existence of different (coherently related) viewpoints of the universe as a whole.

(Brown 2005, p.14)

Of course, Barbour's description is easy enough to understand when material events are taken to have some kind of (pseudo-)metrical structure. But what sense can be made of it if some such structure cannot be assumed, so that it remains to be 
accounted for by the symmetries of the dynamical laws? Granted, questions of this sort aren't Brown's primary focus, but it is clear that Brown must be assuming at least some kind of primitive ordering or arrangement to the world's material events. Indeed, it is as if Brown's material events have all the sub-metrical continuity structure of a differentiable manifold, but not any metrical structure. Thus it would be fair to say that Brown's fundamental metaphysics seems to be a collection of material events with some particular topological, or perhaps even differentiable structure. And in fact, Norton cites personal correspondence that supports this.

In the process of the refereeing of an earlier version of this note, Harvey Brown has assured me that (Brown and Pooley 2006) and (Brown 2005, especially ch.2) presumed the existence of a manifold of spacetime events with coordinate systems.

(2008, p.829)

From this we see that Pooley's reminder above (p5) is apt: the dynamical approach to special relativity needn't account for all geometrical structure by an appeal to the symmetries of the dynamical laws, and indeed Brown seems to have had in mind a primitive sub-metrical structure all along. The dynamical approach's arrow of explanation from dynamics to geometry can then be understood along the lines above: as an application of Einstein's practical geometry to a topological (or differentiable) space of field values, which finds Minkowski geometry to supervene upon the symmetries of the best-system dynamical laws.

\section{Conclusions}

In the sections above, it has been argued that the dynamical approach to special relativity involves explicit explanatory claims - as well as implicit ontological and 
ideological claims - that give it a unique position in the spectrum of traditional approaches to spacetime geometry. The explanatory, ontological, and ideological claims can be satisfied when a primitive sub-metrical structure is ascribed to material bodies in such a way that, given the range of coordinatizations respecting that sub-metrical structure, the set in terms of which are written the best-system dynamical laws are related to one another by elements of the Poincaré group. ${ }^{23}$

If fact, this form of practical geometry has appeared in recent commentaries on Brown's work. For example, it was mentioned above that Norton acknowledges the possibility of taking a topologically structured spacetime as primitive, although no comments are made as to whether that topological structure could instead be assigned to the material objects themselves. ${ }^{24} \mathrm{~A}$ similar suggestion has also been made by Nick Huggett.

One possibility is that unlike geometry, topology is fundamental [... The] topology of the fields plus point coincidences do not determine the metrical properties, so there is additional work to be done by the laws, along the lines sketched above. (2009, p.418)

${ }^{23}$ Following on from footnote 3 , the dynamical approach would also be compatible with a form of ontological substantivalism, according to which there exists a substantival spacetime manifold with sub-metrical structure, from which the inhabiting material objects inherit their own. Minkowski geometry could still supervene along the lines above. Some of Brown's claims about geometry appear open to interpretation along these lines (Brown 2005, p.9, p.100, p.133, p.142), although there is nothing to suggest that Brown actually has this in mind.

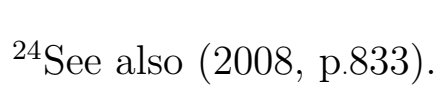


And more recently, Oliver Pooley has suggested something similar $2013, \S 6.3 .2$; 2014 §6.2). The common idea is that the constructivist project can be carried out by considering a material world with sub-metrical spatiotemporal structure, and allowing the best-systems dynamical laws to be written in terms of some geometrical structure that supervenes upon those laws' being best. 


\section{References}

Balashov, Yuri and Janssen, Michel (2003, June). Review: Presentism and relativity. British Journal for the Philosophy of Science, 54(2), 327-46. Reviewed works: The tensed theory of time: A critical examination, by William Lane Craig.

Barbour, Julian (1982). Relational concepts of space and time. The British Journal for the Philosophy of Science, 33(3), 251-274.

Brown, Harvey (1997). On the role of special relativity in general relativity. International Studies in the Philosophy of Science, 11(1), 67-81.

Brown, Harvey (2005). Physical relativity: Space-time structure from a dynamical perspective. Oxford University Press.

Brown, Harvey and Pooley, Oliver (2001). The origin of the spacetime metric: Bell's 'Lorentzian pedagogy' and its significance in general relativity. In Physics meets philosophy at the Planck scale: Contemporary theories in quantum gravity (ed. C. Callender and N. Huggett), Chapter 11, pp. 256-272. Cambridge University Press.

Brown, Harvey and Pooley, Oliver (2006). Minkowski space-time: A glorious nonentity. In The Ontology of Spacetime (ed. D. Dieks), Volume 1 of Philosophy and Foundations of Physics, pp. 67-89. International Society for the Advanced Study of Spacetime: Elsevier.

Dieks, Dennis (2009). Understanding in physics: Bottom-up versus top-down. In Scientific Understanding: Philosophical Perspectives (ed. K. E. Henk de Regt, Sabina Leonelli), pp. 230-48. University of Pittsburgh Press. 
Dorato, Mauro and Felline, Laura (2010). Structural explanations in Minkowski spacetime: Which account of models? In Space, Time, and Spacetime (ed. V. Petkov), Volume 167, Part 2 of Fundamental Theories of Physics, pp. 193-207. Springer.

Earman, John (1989). World enough and space-time: Absolute versus relational theories of space and time. MIT Press.

Einstein, Albert (1905). Zur elektrodynamik bewegter körper. Annalen der Physik, 322(10), 891-921. Note: all quotations and page numbers are taken from the English translation (Perrett and Jeffery 1952). Other English translations are provided by Stachel (1998, pp.123-60) and Miller (1981, pp.392-415).

Einstein, Albert (1921). Geometrie und erfahrung: Erweiterte fassung des festvortrages gehalten an der preussischen akademie der wissenschaften zu berlin, am 27 Januar 1921. Berlin: Julius Springer. Note: all quotations and page numbers are taken from the English translation (Einstein 1954).

Einstein, Albert (1924). Review of Alfred C. Elsbach, Kant und Einstein. Deutsche Literaturzeitung, 1(24), 1685-1692. Note: all quotations and page numbers are taken from the English translation, forthcoming in Vol. 14 of the Einstein Papers Project.

Einstein, Albert (1925). Nichteuklidische geometrie und physik [Non-euclidean geometry and physics]. Die Neue Rundschau, 36(1), 16-20. Translation forthcoming in Vol. 14 of Einstein Papers Project.

Einstein, Albert (1949a). Autobiographical notes. In Albert Einstein: Philosopher- 
scientist (1st edn) (ed. P. Schilpp), Volume VII of The Library of Living Philosophers. Cambridge University Press.

Einstein, Albert (1949b). Remarks to the essays appearing in this collective volume. See Schilpp (1949), Chapter III, pp. 663-687.

Einstein, Albert (1954). Geometry and experience. In Ideas and opinions (ed. C. Seelig), pp. 232-246. Crown Publishers, Inc. Originally a lecture before the Prussian Academy of Sciences, January 27, 1921. First printed as (Einstein 1921).

Felline, Laura (2011, December). Scientific explanation between principle and constructive theories. Philosophy of Science, 78(5), 989-1000.

Friedman, Michael (2002). Geometry as a branch of physics: Background and context for Einstein's 'geometry and experience'. In Reading natural philosophy: Essays in the history and philosophy of science (ed. D. Malament), pp. 368. Chicago: Open Court.

Frisch, Mathias (2011, August). Principle or constructive relativity. Studies In History and Philosophy of Science Part B: Studies In History and Philosophy of Modern Physics, 42(3), 176-183.

Geroch, Robert (1978). General relativity from A to B. University of Chicago Press.

Giovanelli, Marco (2012, December). Talking at cross-purposes: How Einstein and logical positivists never agreed on what they were disagreeing about. Synthese, Published Online, 1-45.

Hempel, Carl (1945, January). Geometry and empirical science. The American Mathematical Monthly, 52(1), 7-17. 
Hilbert, David (1971). Foundations of Geometry (2nd English edn). Open Court: La Salle, Illinois. Translated by Leo Unger from the 10th german edition of Grundlagen der Geometrie. Revised and enlarged by Paul Bernays.

Howard, Don (2014). Einstein and the development of twentieth-century philosophy of science. Forthcoming in The Cambridge Companion to Einstein.

Huggett, Nick (2006, January). The regularity account of relational spacetime. Mind, 115(457), 41-73.

Huggett, Nick (2009, July). Essay review: Physical Relativity and Understanding Space-Time. Philosophy of Science, 36(3), 404-22.

Janssen, Michel (2009). Drawing the line between kinematics and dynamics in Special Relativity. Studies In History and Philosophy of Science Part B: Studies In History and Philosophy of Modern Physics, 40(1), 26-52.

Lange, Marc (2011, September). How to explain the Lorentz transformations. (Unpublished manuscript; work in progress.).

Maudlin, Tim (1993, June). Buckets of water and waves of space: Why spacetime is probably a substance. Philosophy of Science, 60(2), 183-203.

Miller, Arthur (1981). Albert Einstein's special theory of relativity: Emergence (1905) and early interpretation (1905-1911). Addison-Wesley Publishing Company, Inc.

Nerlich, Graham (2013). Einstein's genie: Spacetime out of the bottle, Chapter 5: In SR, why does a rod contract?, pp. 75-98. Minkowski Institute Press.

Norton, John D. (2008). Why constructive relativity fails. The British Journal for the Philosophy of Science, 59(4), 821-834. 
Perrett, W. and Jeffery, G. B. (ed.) ([1905] 1952). On the electrodynamics of moving bodies, Chapter 3, pp. 35-65. New York: Dover Publications, Inc., New York. An unabridged and unaltered republication of the translation first published by Methuen and Company, Ltd. in 1923.

Poincaré, Henri (1898, October). On the foundations of geometry. The Monist, IX(1), 1-43.

Poincaré, Henri (1952 [1905]). Experiment and geometry, Chapter V. Dover Publications, Inc.

Pooley, Oliver (2013, January). Substantivalist and relationalist approaches to spacetime. In The Oxford Handbook of Philosophy of Physics (ed. R. Batterman), Chapter 15, pp. 522-586. Oxford University Press.

Pooley, Oliver (2014). The reality of spacetime. Oxford University Press. (Unpublished manuscript; work in progress.).

Quine, W.V. (1951, January). Ontology and ideology. Philosophical Studies, 2(1), $11-15$.

Reichenbach, Hans (1949). The philosophical significance of the theory of relativity. See Schilpp (1949), Chapter 10, pp. 289-311.

Reichenbach, Hans (1958). The philosophy of space and time. Dover Publications. Translation of Philosophie der Raum-Zeit-Lehre (1928).

Robb, Alfred (1914). A theory of time and space. Cambridge University Press.

Robb, Alfred (1936). The geometry of time and space. Cambridge University Press. 
Schilpp, Paul (ed.) (1949). Albert Einstein: Philosopher-scientist (1 edn), Volume VII of The Library of Living Philosophers. Cambridge University Press, Evanston, Illinois.

Skow, Bradford (2006, May). Physical relativity [book review]: Space-time structure from a dynamical perspective. In Notre Dame Philosophical Reviews. University of Notre Dame.

Stachel, John (ed.) (1998). Einstein's miraculous year. Princeton University Press. van Camp, Wesley (2011, December). On kinematic versus dynamic approaches to Special Relativity. Philosophy of Science, 78(5), 1097-1107.

Winnie, John (1977). The causal theory of space-time. In Foundations of Space-Time Theories (ed. J. Earman, C. Glymour, and J. Stachel), Volume VIII of Minnesota Studies in the Philosophy of Science, Chapter 7, pp. 134-205. University of Minnesota Press.

Zeeman, Erik (1964, April). Causality implies the Lorentz group. Journal of Mathematical Physics, 5(4), 490-493. 